\title{
Anabases
}

ANABASES Traditions et réceptions de l'Antiquité

$21 \mid 2015$

Varia

\section{Antiquités parallèles (2). Femmes antiques}

\section{Claude Aziza}

\section{(2) OpenEdition}

Journals

Édition électronique

URL : http://journals.openedition.org/anabases/5329

DOI : 10.4000/anabases.5329

ISSN : 2256-9421

\section{Éditeur}

E.R.A.S.M.E.

\section{Édition imprimée}

Date de publication : 1 avril 2015

Pagination : 223-227

ISSN : 1774-4296

\section{Référence électronique}

Claude Aziza, «Antiquités parallèles (2). Femmes antiques », Anabases [En ligne], 21 | 2015, mis en ligne le 01 avril 2018, consulté le 20 octobre 2019. URL : http://journals.openedition.org/anabases/ 5329 ; DOI : 10.4000/anabases.5329 


\section{Antiquités parallèles (2) Femmes antiques}

Claude AzizA

«La femme antique est l'avenir de l'homme moderne» Sigmund Freud.

a chronique précédente avait pour héros un Athénien subtil, Thémistocle, un
patriarche inquiet, Noé et un héros bodybuildé, Hercule. Celle-ci mettra en
valeur quelques héroïnes antiques.

\section{Quand les Dieux s'en mêlent...}

On commencera par Bethsabée. On connaît l'histoire: David, devenu, après Saül, le deuxième roi d'Israël, est tombé amoureux de l'épouse d'un de ses capitaines, Urie, qu'il envoie se faire tuer en première ligne. L’opéra, la peinture, la littérature ont glosé sur cet épisode somme toute peu glorieux qui voit la passion triompher de la justice et attirer les foudres jéhoviennes sur les amants. Le cinéma a donné de l'histoire plusieurs versions. Les deux dernières ont choisi des tonalités différentes. La plus récente, Le Roi David de B. Beresford (I985), mêle assez habilement la romance amoureuse et le pouvoir politique face au pouvoir religieux, en donnant à la danse devant l'Arche une interprétation assez inhabituelle qui va dans ce sens. Hélas, le film sorti en vHs n'est toujours pas disponible en DVD. Plus conventionnel, plus sage, plus hollywoodien, le Davidet Bethsabée d'H. King (I95i) ne manque pourtant pas d'allure. Il est sorti en DvD.

Mais dans l'histoire de David, on oublie souvent un élément important: ses rapports ambigus avec Saül et Jonathan, le fils de celui-ci. Pour A. Gide, la mort de Saül (Saül, ı898-ıgo3) est la conséquence de la passion qu'il voue à son poète et son dépit devant la liaison qu'il a avec Jonathan. T.Burnett Swan (r928-ı976) 
dans Plus grands sont les héros (1974, TR. Les Moutons électriques, Hélios, I4, 2014) a tenté de creuser ce qui unit les deux jeunes hommes, en donnant à son récit des couleurs parfois surnaturelles. Or l'auteur, peu connu en France, devrait intéresser tout amateur d'antiquité à laquelle il a consacré deux trilogies passionnantes. La première a pour héros le Minotaure et on la trouve aujourd'hui regroupée en un seul volume chez Folio SF, n 204 (I. Le Labyrinthe du Minotaure; 2. La Forêt du Minotaure; 3. Le Jour du Minotaure). La seconde, à mes yeux plus intéressante, se nomme Le Cycle du Latium et est centrée sur les débuts de Rome en leur donnant des couleurs nouvelles. Le premier volume, Le Phénix vert (Points fantasy, $n^{\circ} \mathrm{P}$ (67o), nous montre un Énée barbare et brutal accoster sur les rives d'un monde où règnent encore faunes et dryades qui devront l'affronter. Dans le deuxième, Le Peuple de la mer (Id., n $\left.{ }^{\circ} \mathrm{P}_{\mathrm{i}} 29\right)$, prend place l'épisode didonien. Le troisième, La Dame des abeilles (Id., n ${ }^{\circ} \mathrm{P}$ (767) a pour héros les fameux jumeaux qui, alliés aux dryades et aux faunes (les revoilà), vont conquérir Albe. Ce n'est qu'après la mort du pacifique et doux Remus que pourra commencer - sur le fratricide que l'on sait - la fondation de Rome. Cette alliance entre SF et Antiquité n'est pas nouvelle. On en veut pour preuve deux recueils récents de nouvelles. Le premier, Fragments d'une fantasy antique, est paru chez Mnémos en 2012, le second, Dimension antiquité, chez Rivière blanche en 20I4. Dans les deux cas, des romanciers, français et étrangers, prennent pour cadre le monde antique. Plus étonnant, cependant, le récit de l'installation d'Énée en terre latine et de sa rencontre avec celle qui deviendra son épouse, Lavinie. Lavinia est un roman d'U. Le Guin (2008, TR. L'Atalante, 20Io), une grande dame de la SF, peut-être la plus grande, dont l'œuvre a été surtout consacrée à la défense et à l'illustration de la condition féminine à travers les mondes de la galaxie. Son chef-d'œuvre reste La Main gauche de la Nuit (1969, Pocket SF, nْ 5ıgI, mais peut-être plus disponible). La planète Gethen-Nivôse, située aux confins de l'univers, à une centaine d'années-lumière de la Terre, est un monde sans sexe défini: ses habitants sont, en suivant un cycle d'ovulation, successivement hommes et femmes. Ce qui trouble profondément le héros du roman mais engendre un monde pacifique et hautement civilisé. Ce qui n'est pas le cas de celui qui voit Grecs et Troyens s'affronter...

\section{Remue-ménage à Troie}

Deux romans pour raconter la Belle Hélène. Romans assez médiocres, il faut bien l'avouer. Le premier de M. Georges, Hélène de Troie, en 2 volumes (Le Livre de poche, nos 3i214 et 3i215) est d'une honnête mais plate facture. Le second, de F. Petrizzo, Mémoires d'une catin (20I0, trad. Michel Lafon, 2010) se vend sur un titre inutilement provocateur et sur une intrigue de bazar. Mieux vaut se pencher sur les héros grecs. Achille est au cœur d'un très poétique récit de M. Miller, Le Chant d'Achille (20I3, TR. Rue Fromentin, 20I4) et Ulysse d'un beau roman de V. Manfredi : 
Odysseus. Les Rêves d'Ulysse (2014, TR. J.-C. Lattès, 2014), dont on attend la suite. Autre séductrice mais, peut-être, malgré elle, Salomé.

\section{La danse des 7 toiles}

La sortie de 2 DVD consacrés à Salomé (C. Bryant, I923; W. Dieterle, I952) m'incite à explorer le sort cinématographique de la fameuse danseuse. L'épisode de Salomé a été rapporté par deux évangélistes: Matthieu (I4, I-I2) et Marc (6, I4-28). Il est absent de Luc qui, en 3, I9-20, mentionne seulement l'emprisonnement de Jean le Baptiste par Hérode Antipas. Tout comme Josèphe dans les Antiquités Judaïques, XVIII, 7. On connaît sa fortune littéraire (Flaubert, Laforgue, Mallarmé, Wilde et bien d'autres encore), picturale (Moreau) et musicale (Massenet, Schmitt, Strauss). Mais on ignore, en général, sa richesse cinématographique. Entre Igo2 et 2002, pas moins de 25 adaptations (dont 16 pour le muet) qui à partir des Évangiles, qui à partir de la pièce - écrite en français (on conseillera la version bilingue chez GF, $\mathrm{n}^{\circ} 649$, avec la traduction anglaise d'A.Douglas, I894) - d'Oscar Wilde (I894, mais représentée le ı2 février I8g6 au Théâtre de l'Euvre à Paris, avec Sarah Bernhardt). Sans compter les parodies, les transpositions et les apparitions de l'héroïne dans quelques vies de Jésus, dont Le Roi des Rois, N. Ray, Ig6I, L'Évangile selon saint Matthieu, P. P.Pasolini, I964, Jésus de Nazareth, F.Zeffirelli, I977. Tous trois disponibles en DVD. Passons au détail de sept adaptations.

- La plus Vamp:

Si la première, en Ig02, est allemande, la première réussite est due au grand maitre américain du muet: J.Stuart Blackton, en igo8. Il faudra attendre cependant encore dix ans pour qu'en ıوı J. Gordon Edwards, s'inspirant de Wilde et de Strauss, fasse incarner Salomé par la première vamp américaine (et mondiale), Theda Bara (I89o-I955) qui venait de jouer Carmen, Sapho, Juliette, Hélène, Cléopâtre et... Madame du Barry.

- La plus Wildienne (désormais accessible):

En I922, dans des décors et avec des costumes qui évoquent plus l'art nouveau que le Nouveau Testament, Alla Nazimova (I879-1945) évolue avec grâce, le visage blanchi et la perruque claire, dans un film américain de Ch. Bryant. La costumière, Natasha Rambova (seconde épouse de Rudolph Valentino) avait tenté de recréer les costumes dessinés par Aubrey Beardsley pour la première de la pièce de Wilde. L'actrice, aux goûts somptueux et extravagants, s'était ruinée pour financer ce film qui fut un échec. Elle dut, sur les écrans, céder la place à Greta Garbo.

- La plus étrange:

Aucun cinéaste n'osant se mesurer à Ch. Bryant, Ch.Lamont tourna la difficulté en 1945: dans Les Amours de Salomé, il transposait l'histoire dans un décor de western et offrait à la voluptueuse Yvonne de Carlo l'un de ses plus beaux rôles (chercher le film en vHs, chez AlpaMedia). 
- La plus pro (désormais accessible):

Il convenait de revenir à l'antique et de confier le rôle à une authentique danseuse. Ce fut Rita Hayworth en ig52 dans la Salomé de W.Dieterle, tirée d'un roman que le cinéaste avait lui-même écrit sous pseudonyme. On y raconte les amours de Salomé, élevée à la cour de Tibère, et d'un beau centurion romain. Après une somptueuse scène de danse, Salomé, déjà préchrétienne, s'enfuit avec son légionnaire. R. Anderson a tiré une novellisation du film, parue en traduction française en ig53, chez André Martel.

- La plus corrosive:

Les années ig7o virent le retour du mythe traité à la façon « underground ». Dans un film allemand de W. Schroeter (I97I), tiré de Wilde, Salomé se présente comme une icône glaciale. Mais c'est C. Bene, toujours d'après Wilde, en I972, qui donne de la pièce de Wilde une image visuellement délirante et troublante.

- La plus scandaleuse:

On ne pouvait attendre de K. Russel autre chose qu'une version à scandale. Jugez-en: dans un film de ig88, Wilde assiste, dans un lupanar londonien, à une représentation privée de sa pièce. Représentation qui commence par la fameuse danse (chercher le film en vHs chez Delta Vidéo).

- La plus musicale:

La dernière mouture du mythe, la Salomé de C. Saura (2002), existe en DVD. Il s'agit d'un ballet dont l'éblouissante chorégraphie moderne, les jeux de lumière et l'alliance des mouvements de caméra et de ceux des corps sont une véritable transfiguration de la légende.

\section{Victimes et bourreaux}

Elle s'appelait Paulina, elle était originaire de la Narbonnaise et elle devint l'épouse de Sénèque. Triste fut le destin de cette femme intelligente et cultivée qui assista au suicide, imposé par Néron, de son époux, mais fut épargnée par l'empereur. P. de Carolis raconte son histoire dans La Dame du Palatin (Plon, 20II), un beau roman. Il faudra le lire en même temps que Villa du crépuscule de J. Browner (2007, TR. Phébus, 2009) qui raconte la dernière nuit de Pétrone, et que Quitte Rome ou meurs de R. Sardou (2009, XO éditions, Pocket, $n^{\circ}$ I4438) dont l'intrigue se passe à la cour de Néron. On pourra agrémenter le cocktail en visionnant deux versions de Quo Vadis? aujourd'hui disponibles en DvD, celle de M. Le Roy (I95i) et celle de J. Kawalerowicz (200I), sans oublier que la version intégrale (toutes les autres étaient amputées) du roman est disponible aux Belles Lettres (20I0). Ajoutons-y Légions. Les Guerriers de Rome (un film de B. Anderson, 2003) pour le plaisir de voir le jeune Néron sucer avidement le sein maternel!

Elle s'appelait Fabiola, elle naquit sous la plume du cardinal Wiseman, en I854 (la chercher d'occasion en Pocket) et connut un succès mondial. Longtemps 
indisponible, la version d'A. Blasetti (I948) est en DvD. On peut y voir - avec un peu d'attention - quelques fils télégraphiques laissés par mégarde mais surtout assister in live, sur la plage d'Ostie, aux amours de Michèle Morgan (la belle patricienne romaine) et d'Henri Vidal (le beau gladiateur gaulois). On s'en doute, ces festons érotiques ne se trouvaient pas dans le roman.

On ne connaît pas le nom de la concubine d'Augustin, cette femme mystérieuse, mère de son fils Deodatus, qui disparut de la vie du futur saint quand il renonça à vivre dans le péché. Cl. Pujade-Renaud lui rend justice dans un beau et grave roman, Dans l'ombre de la lumière (Actes Sud, 20I3, Babel, n I24I).

Terminons enfin par le destin tragique d'Hypatie, philosophe, astronome et mathématicienne. Mais surtout femme libre que les fanatiques chrétiens de l'Alexandrie $\mathrm{du} \mathrm{Iv}^{\mathrm{e}}$ siècle, conduits par l'évêque Cyrille, assassinèrent avec une cruauté barbare, au nom du Christ. Ainsi donc les victimes étaient devenues bourreaux! A. Amenabar en a fait, en 20Io, un film superbe, vigoureux plaidoyer contre l'intolérance, qui fut en butte à l'hostilité de l'Église d'Espagne et passa quasiment inaperçu en France. Heureusement le DvD est désormais là.

Ah, j'allais oublier Alcibiade! Sa vie scandaleuse est racontée dans un roman d'E.Jourdan, Sans lois ni dieux ( $\mathrm{H}$ et $\left.\mathrm{O}, 2010, \mathrm{n}^{\circ}{ }^{22}\right)$. Mais que vient-il faire ici? En souvenir de François Villon qui - comme tous ses contemporains - le croyait femme... (voir sa Ballade des Dames du temps jadis : “Dites-moi où, n’en quel pays// Est Flora la belle Romaine// Archipiades ne Thaïs// Qui fut sa cousine germaine? »).

\section{Claude Aziza}

Université de la Sorbonne Nouvelle, Paris III

6, square de Port-Royal, 75013 Paris

claude.aziza@laposte.net 